\title{
Écosystème de représentations et apprentissage de la conception
}

\author{
Julie Milovanovic ${ }^{1, *}$, Daniel Siret ${ }^{1}$, Guillaume Moreau ${ }^{2}$ et Francis Miguet $^{1}$ \\ ${ }^{1}$ UMR AAU-CRENAU, École Nationale Supérieure d'Architecture de Nantes, France \\ ${ }^{2}$ UMR AAU-CRENAU, École Centrale Nantes, France
}

\begin{abstract}
Résumé. L'article construit une articulation des notions d'écosystème de représentations, d'apprentissage et de conception. Le modèle descriptif proposé permet d'interroger l'impact d'une altération de l'écosystème de représentations sur l'apprentissage de la conception en studio de projet. Deux écosystèmes utilisant des techniques de Réalité Virtuelle pour favoriser l'immersion dans le projet sont analysés. Les implications sur les processus d'apprentissage de la conception sont décrites par rapport au modèle descriptif proposé.
\end{abstract}

Mots-Clés. Apprentissage, Écosystème de représentations, Conception, Réalité Virtuelle.

\begin{abstract}
This article develops an articulation of three notions: representational ecosystem, learning and design. The proposed descriptive model gives a framework to question the effect of an alteration of the representational ecosystem on design learning in design studios. A study of two representational ecosystems using Virtual Reality techniques to enhance the feeling of immersion in design representations is described. Their implications on design learning are put forward in the light of the proposed descriptive framework.
\end{abstract}

Keywords. Learning, Representational ecosystem, Design, Virtual Reality.

\section{Introduction}

À travers la notion de designerly ways of knowing, Cross (1982) exprime la spécificité de la conception comme mode de pensée, de faire et de connaître. La recherche en conception a généré de multiples théories et modèles de la conception (Cross, 2007). Cette activité est décrite comme une activité sociale (Bucciarelli, 2001 ; Callon, 1996), dirigée par un processus de réflexion-dans-l'action (Schön, 1983), où une idée ou « concept » peut

\footnotetext{
*Auteur correspondant : julie.milovanovic@crenau.archi.fr
} 
être fixé pour réduire l'ensemble de possibilités (Darke, 1979), et où l'espace du problème de conception et l'espace de la solution co-évoluent (Maher et Poon, 1996) en fonction de l'expertise du concepteur (Dorst et Cross, 2001). Par l'expérience, les concepteurs développent des schémas de conception (Lawson, 2004), référencés par d'autres auteurs comme répertoires de conception (Schön, 1983), ou prototypes de conception (Gero 1990). L'activité de conception est une forme de conversation réflexive entre le concepteur et l'ensemble des représentations externes du projet (Schön, 1992). La notion d'écosystème de représentations (Dorta et al., 2016a ; Dorta et Kinayoglu, 2014), intègre l'ensemble des représentations externes du projet telles que les croquis diagrammes, plans, coupes, maquettes physiques, maquettes numériques, simulations et animations. Il est considéré comme un acteur de la conception dans le sens où il sert de support à la conversation réflexive (Schön, 1992) et à la simulation graphique (Lebahar, 1983).

L'objet d'apprentissage au cœur du curriculum de la formation en école d'architecture est l'ensemble des modes de pensée, de faire et de connaître du concepteur. Pour les étudiants, l'apprentissage passe par la construction de schémas cognitifs, à travers les expériences de conception. La notion d'apprentissage de la conception est liée, dans la littérature, aux notions d'apprentissage par le faire et de mentorat (Cross, 2006 ; Lawson et Dorst, 2009 ; Schön, 1985). Le studio de projet est l'un des lieux de cet apprentissage où l'étudiant apprend par le faire. L'objectif y est la production d'un projet architectural, qui répond aux contraintes posées par l'enseignant. La critique de projet hebdomadaire est l'occasion pour les étudiants de présenter leur projet et d'avoir un retour de leurs enseignants sur leur avancement, ce qui en fait un moment clé. L'altération de l'écosystème de représentations, en y incluant des représentations immersives grâce aux techniques de la Réalité Virtuelle (RV) ouvre de nouvelles potentialités en termes d'apprentissage.

Dans la première partie de cet article, nous proposons un modèle descriptif de l'apprentissage de la conception en studio de projet en synthétisant des cadres théoriques existants : le modèle de conception située de Gero et Kannengiesser (2004) et le cycle d'apprentissage expérientiel de Kolb (1984). L'enjeu est de pouvoir définir, à travers ce modèle, les implications induites par l'utilisation de représentations immersives sur les processus d'apprentissage de la conception. Dans la deuxième partie de l'article, deux cas sont étudiés : le HYVE 3D (Dorta et al., 2016b), utilisé comme outil pédagogique de support de la critique en studio de codesign à l'Université de Montréal ; et le système CAP VR (Angulo, 2015), exploité dans le cadre d'un studio de projet d'architecture à Ball State University. Nos analyses se basent sur un corpus d'articles décrivant l'utilisation de ces écosystèmes de représentations pour le studio (Angulo, 2015 ; Angulo et de Velasco, 2015 ; Dorta et al., 2012 ; Dorta et al., 2016a ; Dorta et al., 2016b), des observations directes (pour le HYVE-3D) et des échanges avec les enseignants ayant mis en place ces écosystèmes immersifs pour leurs enseignements.

\section{Vers un modèle descriptif d'apprentissage de la conception}

\subsection{Apprentissage en studio de projet}

L'apprentissage s'apparente à un changement du comportement de l'individu, dans une situation particulière, et qui est lié à ses expériences (Hergenhahn et Olson, 1997 ; Phillips et Soltis, 2009). Le format pédagogique du studio de projet en école d'architecture est défini par des caractéristiques proches des principes des théories de l'apprentissage constructiviste et expérientiel que nous allons examiner maintenant.

Du point de vue constructiviste, l'apprentissage est séquentiel, ce qui signifie qu'il y a un développement progressif des structures cognitives de l'apprenant. Piaget (1975), l'une des 
figures majeures du constructivisme, explique que nous possédons un ensemble réduit de schémas innés qui expriment notre potentiel à agir sur notre environnement. La combinaison de tous ces schémas forme notre structure cognitive. Celle-ci change au fil du temps, de même que nos actions potentielles sur notre environnement, puisque la structure cognitive affecte la manière dont nous nous comportons. Piaget définit deux processus relatifs au développement de la structure cognitive : l'assimilation et l'accommodation. L'assimilation correspond à l'association entre la structure cognitive et l'environnement, que l'on peut représenter par l'action de connaître ou reconnaître. L'accommodation déclenche un changement dans la structure cognitive qui se produit lorsqu'un événement dans l'environnement ne correspond pas exactement aux schémas de la structure cognitive actuelle. Lorsque l'événement n'est pas reconnu, il crée un déséquilibre dans les structures cognitives, qui vont s'adapter pour trouver un nouvel équilibre. Piaget a formalisé l'importance de la relation individuelle entre les enseignants et les étudiants afin que l'information présentée aux étudiants soit appropriée à l'état actuel de leur structure cognitive. Le modèle de l'apprentissage expérientiel de Kolb (Kolb et Kolb, 2005 ; Kolb, 1984), s'appuyant sur les travaux de Piaget (1975) et de Dewey (1938), représente un cycle d'apprentissage en quatre temps liés à l'expérience: l'expérience concrète (EC), l'observation réflexive (OR), l'abstraction conceptuelle (AC) et l'expérimentation active (EA). L'apprentissage passe par une transformation cyclique de l'expérience, qui peut entrainer un ajustement des structures cognitives de l'apprenant (accommodation). Ce modèle d'apprentissage s'adapte aux moments du studio de projet et offre un cadre théorique pour développer la notion d'apprentissage de la conception dans le cas du studio de projet (Parnell et al. 2007 ; Khorshidifard, 2011).

Le format du studio de projet peut être calqué sur l'approche constructiviste et expérientielle de l'apprentissage : les étudiants sont confrontés à une tâche qu'ils ne peuvent pas complètement résoudre (concevoir un projet en fonction des exigences de conception), provoquant un déséquilibre dans leur structure cognitive, qu'ils ajustent par leurs interactions avec leur environnement social par une itération de phase d'apprentissage. L'enseignant joue un rôle majeur en accompagnant les étudiants pour qu'ils assimilent de nouvelles informations sur la conception. Les enseignants prennent une position de coach en poussant les étudiants à réfléchir sur la solution qu'ils proposent plus qu'à leur donner une solution toute faite (Adams et al., 2016). Les sessions critiques informelles rythment la temporalité du studio et se formalisent par une discussion en tête-à-tête entre les enseignants et les étudiants. Dans cette situation, les enseignants sont en mesure d'évaluer les capacités réelles des étudiants, afin de leur fournir un retour adéquat pour qu'ils poursuivent leur activité de conception.

\subsection{Modèle de conception située}

L'apprentissage de la conception est étroitement lié à l'activité de conception en soi car les étudiants apprennent par le faire (Cross, 2006 ; Lawson et Dorst, 2009 ; Schön, 1983, 1985). Pour cela, nous proposons d'adapter un modèle de conception (l'ontologie Function Behavior Structure, FBS, située de Gero, 1990 ; Gero et Kannengiesser, 2004) à la situation du studio de projet (alternance de sessions de conception autonome et de critique de projet), et aux phases d'apprentissage expérientiel de Kolb (1984).

L'ontologie FBS située (Gero, 1990 ; Gero et Kannengiesser, 2004) synthétise, en un modèle descriptif, les différentes caractéristiques de la conception que nous avons évoquées. Dans ce modèle, un seul concepteur est représenté, et nous en proposons une extension avec deux concepteurs qui s'adaptera à la situation du studio (figure 1). L'ontologie décrit quatre processus cognitifs de conception, propres à chaque concepteur, qui illustrent une interaction entre trois mondes. Le monde externe est composé des 
représentations externes du projet, partagées par les deux concepteurs. Le monde interprété représente la construction interne du monde par rapport à l'expérience sensorielle, les perceptions et concepts acquis de chaque concepteur. Le monde attendu contient les actions imaginées de la conception et la prédiction de leurs effets. Il inclut l'ensemble des solutions potentielles imaginées par le concepteur et se situe à l'intérieur du monde interprété. Dans chacun de ces mondes, le projet est représenté par sa fonction $(\mathrm{F})$, son comportement (Be ou Bs) et sa structure (S) (cf. ontologie FBS Gero (1990)). Seulement dans le monde externe s'y ajoute les descriptions (D) qui sont les représentations graphiques ou numériques du projet et les contraintes (R) liées au programme ou aux exigences du client.

Quatre processus expriment les relations entre ces trois mondes. L'interprétation représente l'interaction entre le monde externe et le monde interprété (processus 1, figure 1). Elle transforme des variables du monde externe dans le monde perçu par le spectre de la sensation, la perception et les concepts acquis. La structure actuelle de la représentation interprétée du projet donne un filtre de lecture de la situation présente. À travers cette structure, le concepteur voit l'état actuel du projet. La notion de mémoire constructive illustre un processus d'interaction entre concepts interprétés par rapport à la situation actuelle et concepts interprétés basés sur l'expérience passée (processus 2, figure 1). S'il y a un déséquilibre entre la structure interprétée acquise et la structure interprétée par rapport au monde externe actuel, il peut y avoir un ajustement de la structure initiale interprétée, qui est une forme d'apprentissage (cf. Lawson et Dorst (2009) sur la conception comme apprentissage). Le processus de concentration exprime une implication des variables du monde interprété dans le monde attendu (processus 3, figure 1). Il suggère une action dans le monde externe. Enfin, l'action, verbale, graphique ou gestuelle, implique un changement dans le monde externe par rapport aux attentes définies dans le monde attendu (processus 4, figure 1).

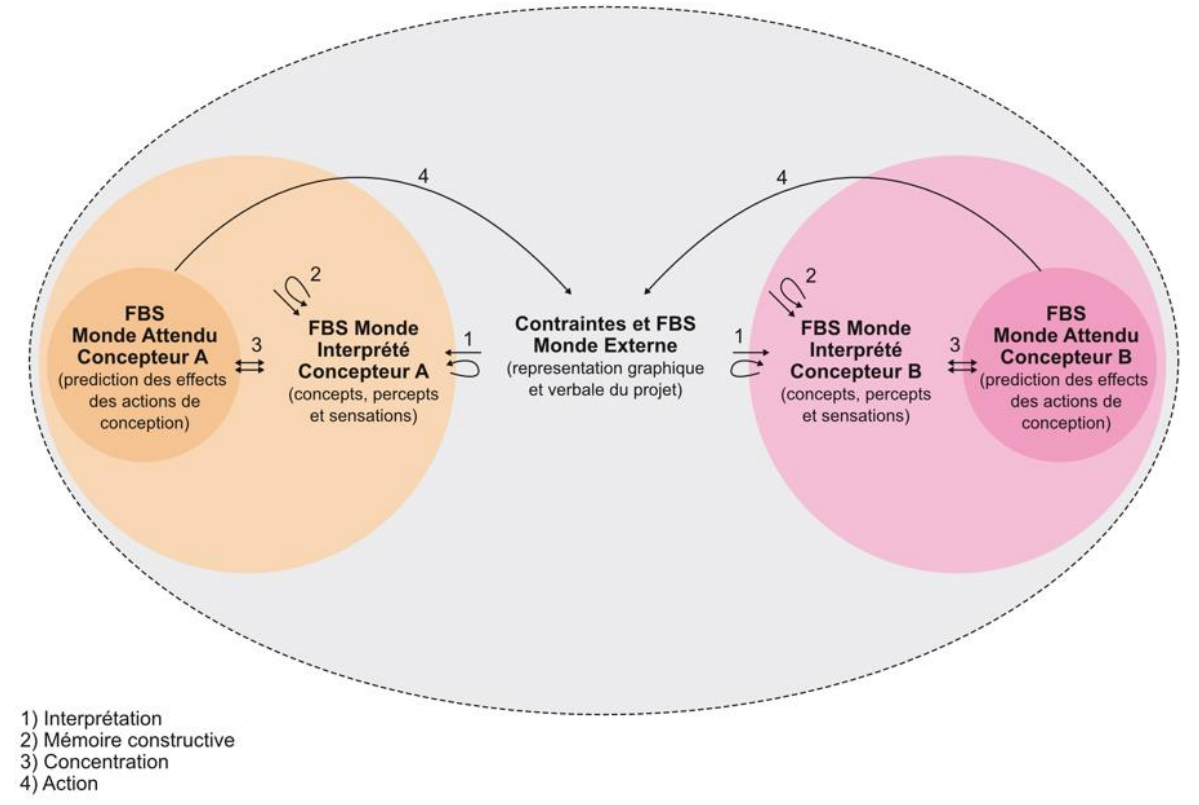

Figure 1. Activité de conception située (source : Gero 1990 ; Gero et Kannengiesser, 2004). 


\subsection{Proposition d'un modèle d'apprentissage de la conception en studio}

L'activité de conception, dans le cadre du studio, se distingue par deux moments : des sessions de conception où l'étudiant travaille en autonomie et des sessions de tutorat, en présentiel, où étudiants et enseignants échangent sur le projet. Le premier moment est dominé par l'expérimentation active et l'expérience concrète, alors que le second s'associe principalement à de l'observation réflexive et l'abstraction conceptuelle (figure 2). Lors de la critique, l'expérience de conception est explicitée à travers la présentation du projet. La réflexion et l'échange construit autour du projet établit une nouvelle base de concepts pour la suite du travail. L'expérimentation active se traduit par l'expérimentation de ces concepts dans le projet, qui mène à une expérience concrète où l'étudiant a une nouvelle expérience située de l'activité de conception.

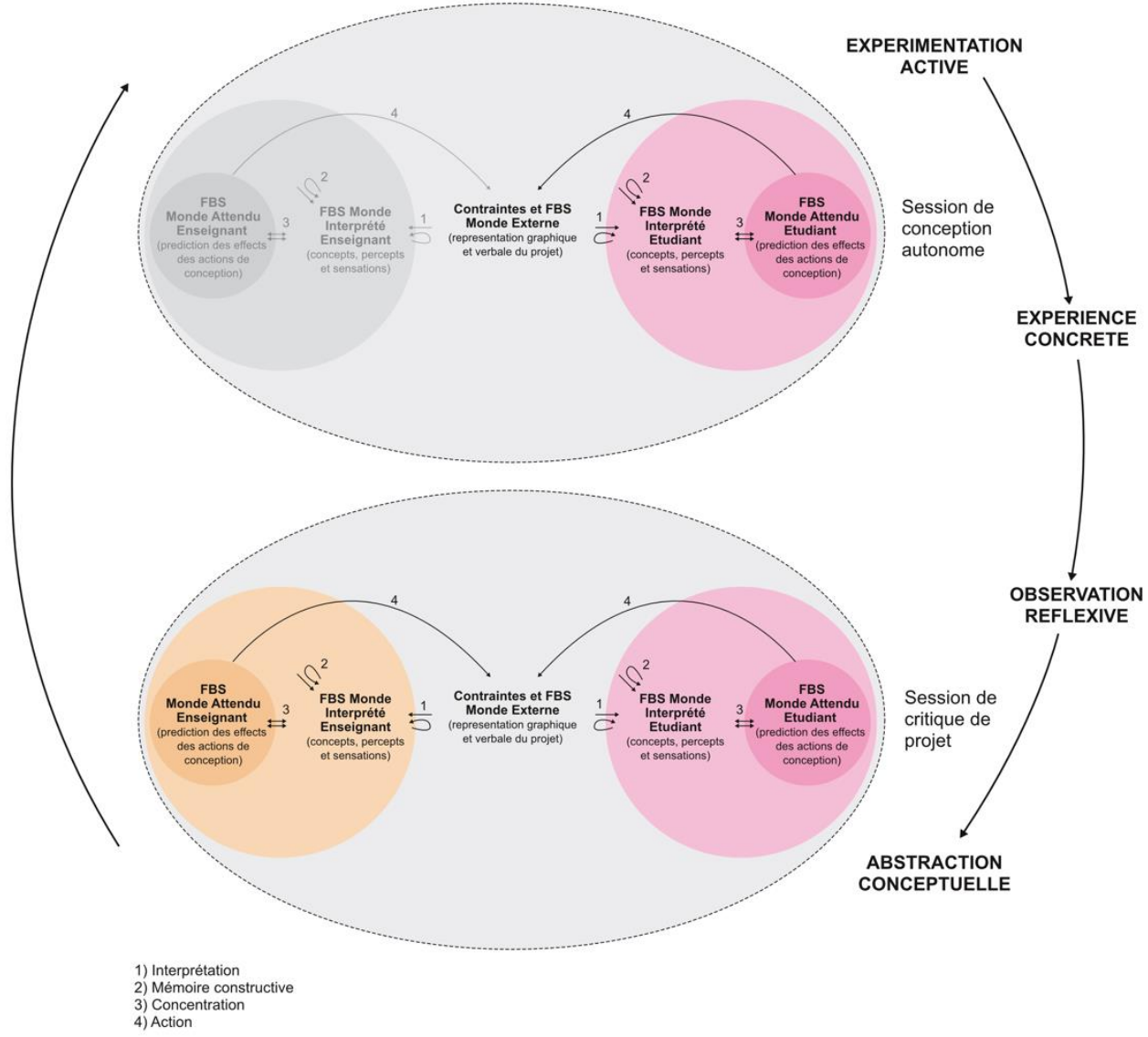

Figure 2. Proposition d'un modèle d'apprentissage situé de la conception.

\section{3. Écosystème de représentations immersives}

Les représentations immersives utilisant la Réalité Virtuelle (RV) sont de plus en plus présentes dans l'activité de conception et dans l'enseignement de la conception. Nous pouvons distinguer trois types de support d'environnement en RV : l'utilisation d'un casque HMD, d'un CAVE (Cruz-Neira et al., 1992) ou d'un écran immersif. Ils offrent chacun des potentiels différents: conception et esquisse immersive, augmentation de l'expérience sensible et évaluation des qualités spatiales, visualisations de simulations, collaboration 
délocalisée ou encore conception à échelle réelle (Milovanovic et al., 2017). Ces dispositifs de RV altèrent l'écosystème de représentations habituel de la conception architecturale. Leur utilisation dans le cadre du studio de projet impacte le processus d'apprentissage de la conception. Pour comprendre cet effet, nous proposons une analyse de deux types de systèmes immersifs utilisés en studio de projet : le HYVE 3D (Dorta et al., 2016b) et le casque immersif CAP VR (Angulo, 2015). Dans le HYVE-3D, le curseur 3D permet la navigation et le dessin, l'environnement virtuel (EV) est projeté en perspective avec un effet trompe l'œil sur un écran courbe (figure 3a). Pour le dispositif CAP VR, l'utilisateur porte un casque « traqué » et peut se déplacer dans un espace réel limité pour naviguer dans l'EV (figure 3b). Une baguette (6 DOF) est également "traquée » et permet un déplacement à plusieurs vitesses. Le point de vue de l'utilisateur portant le casque est projeté sur un écran vertical pour que les autres participants puissent partager son expérience.

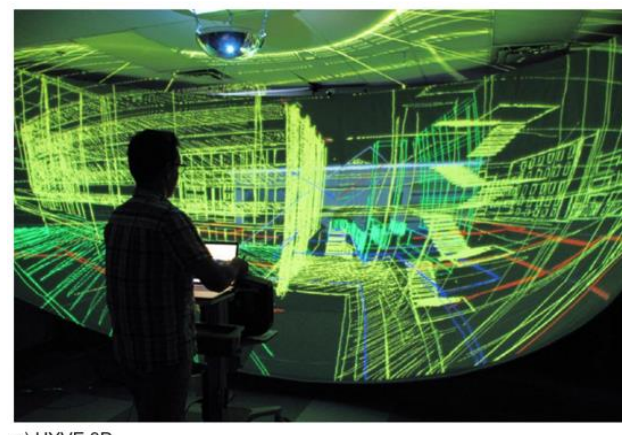

a) HYVE 3D

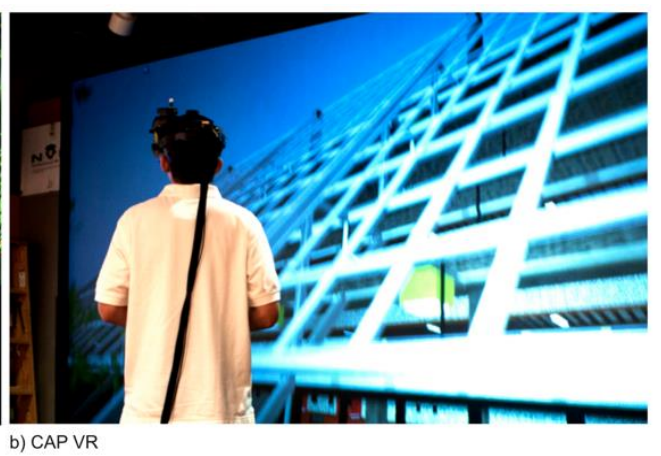

Figure 3. (a) HYVE 3D (source : Dorta et al., 2016b), (b) CAP VR (source : https://capvrenvironment.wordpress.com/).

\subsection{Caractéristiques et implications du CAP VR et du HYVE 3D}

L'écosystème de représentations est contenu dans le monde externe du modèle de conception située (figure 1). Il doit conserver la dimension sociale de la conception en étant un lieu de partage et de collaboration (Dorta et al., 2016a). Quatre caractéristiques le définissent: hybride pour permettre des allers-retours entre analogique et numérique; intégrant des représentations de dimensions et de types multiples (2D, 3D, 4D); comprenant des représentations de différentes échelles, y compris l'échelle 1 (simulation immersive) et permettant le codesign intuitif entre les différents participants (Dorta et al., 2016a). Une cinquième caractéristique importante est sous-entendue dans la notion d'écosystème : la synchronisation de l'ensemble des représentations, donnant une vue complète et unifiée du projet. Nous proposons de synthétiser ces caractéristiques en cinq critères : matérialités, dimensions, échelles, interactions et synchronisation.

Une analyse comparative des deux écosystèmes de représentations que nous avons introduits précédemment nous permet de voir leurs implications sur les processus d'apprentissage représentés en figure 2. Notre étude se base sur un corpus d'articles (Angulo, 2015 ; Angulo et de Velasco, 2015 ; Dorta et al., 2012 ; Dorta et al., 2016a ; Dorta et al., 2016b), un entretien avec A. Angulo qui a mené, sur plusieurs années, un studio de projet à Ball State University utilisant le CAP VR; de nombreux échanges avec T. Dortá sur l'utilisation du HYVE $3 \mathrm{D}$ en studio de codesign et design industriel à l'Université de Montréal ; des observations directes lors d'un atelier intensif (2 jours) avec 5 étudiants de l'ENSA Nantes travaillant sur leur projet dans le HYVE 3D installé à Bordeaux (LID, en collaboration avec S. Cardoso), ainsi qu'un entretien avec ces 5 participants. 
Le HYVE 3D et le CAP VR présentent des caractéristiques différentes concernant les cinq dimensions de l'écosystème de représentations (tableau 1). Le HYVE 3D favorise la collaboration comme l'espace est partagé et l'expérience de l'EV est similaire pour l'ensemble des acteurs (étudiants, enseignants, pairs). Le CAP VR offre deux types d'expérience, celle pour l'utilisateur portant le casque (étudiant) et celle pour les autres participants qui visualisent l'EV sur un écran vertical (enseignants, pairs). La communication entre les participants est altérée dans le CAP VR car le port du casque bloque les interactions visuelles, gestuelles et graphiques, ce qui n'est pas le cas dans le HYVE 3D.

Tableau 1. Caractéristiques du HYVE 3D et CAP VR

\begin{tabular}{|c|c|c|}
\hline & HYVE 3D & CAP VR \\
\hline Matérialités & $\begin{array}{l}\text { Numérique - Analogue (non } \\
\text { synchronisé avec l'EV) }\end{array}$ & $\begin{array}{l}\text { Numérique - Analogue (non inclus dans l'en- } \\
\text { vironnement et non synchronisé avec l'EV) }\end{array}$ \\
\hline \multirow[t]{3}{*}{ Dimensions } & 2D (vue sur le curseur 3D) & 2D (non inclus dans l'EV) \\
\hline & 3D (écran immersif) & 3D (casque et écran vertical) \\
\hline & Animation 4D (écran immersif) & Animation 4D (casque et écran vertical) \\
\hline \multirow[t]{2}{*}{ Échelles } & Échelle 1 (écran immersif) & Échelle 1 (casque et écran vertical) \\
\hline & Échelle dynamique (curseur 3D) & $\begin{array}{l}\text { Échelles autres (non inclus dans } \\
\text { l'environnement) }\end{array}$ \\
\hline Interactions & Graphiques, visuelles et verbales & Verbales \\
\hline Synchronisation & $\begin{array}{l}\text { Représentations 2D et 3D numé- } \\
\text { riques (pas de synchronisation avec } \\
\text { représentation analogue) }\end{array}$ & Pas de synchronisation \\
\hline
\end{tabular}

Le CAP VR a été utilisé durant plusieurs années à Ball State University, dans le cadre de studios de projet d'architecture (Angulo, 2015; Angulo et de Velasco, 2015). Le workflow entre différents outils de conception et de représentation s'est affiné au fil des expériences en studio. Le processus mis en place par les enseignants suit le déroulé suivant : une visite du site est complétée par une introduction au CAP VR où les étudiants parcourent l'EV de leur site de projet. Ensuite les étudiants travaillent sur la proposition d'idées par différents modes: dessins, maquettes physiques, volumétries, intentions d'ambiances et simulation en RV. Les aller-retours entre le dessin et le rendu RV sont faits de manière itérative. Les étudiants ont accès au CAP VR pendant leur session de conception en autonomie et lors des sessions critiques, ce qui implique que le changement de l'écosystème a un effet sur l'ensemble des moments d'expérience du cycle d'apprentissage situé (figure 4a). Lors des sessions autonomes, les étudiants utilisent le CAP VR pour évaluer leur projet, et pendant les sessions critiques, ils l'utilisent comme support de discussion avec enseignants et pairs. Dans cette configuration, l'étudiant porte le casque et les autres participants visualisent le projet sur l'écran vertical. De ce fait, les actions sur le monde externe, lors des sessions de projet sont uniquement verbales quand le CAP VR est utilisé (processus 4, figure 4a).

Le HYVE 3D (Dorta et al., 2016b), anciennement le HIS (Dorta et Pérez, 2006), est utilisé dans le cadre de studios de codesign et de design industriel à l'Université de Montréal (Dorta et al., 2012 ; Dorta et al., 2016b). Cet écosystème de représentations est exploité sur le temps du studio de projet, durant les sessions critiques. L'environnement immersif s'adapte aux documents produits par les étudiants entre les sessions (sketch 2D ou $3 \mathrm{D}$, maquette numérique ou analogue, animation), qui sont partagés entre les participants durant les sessions critiques. Cette flexibilité lui donne la qualité de suivre l'évolution des médiums utilisés sur la temporalité du projet (de l'esquisse numérique $2 \mathrm{D}$ et $3 \mathrm{D}$ à la maquette numérique et aux animations). Dans cette configuration, les processus altérés se situent sur les phases d'observations réflexives et d'abstractions conceptuelles (figure 4b). 


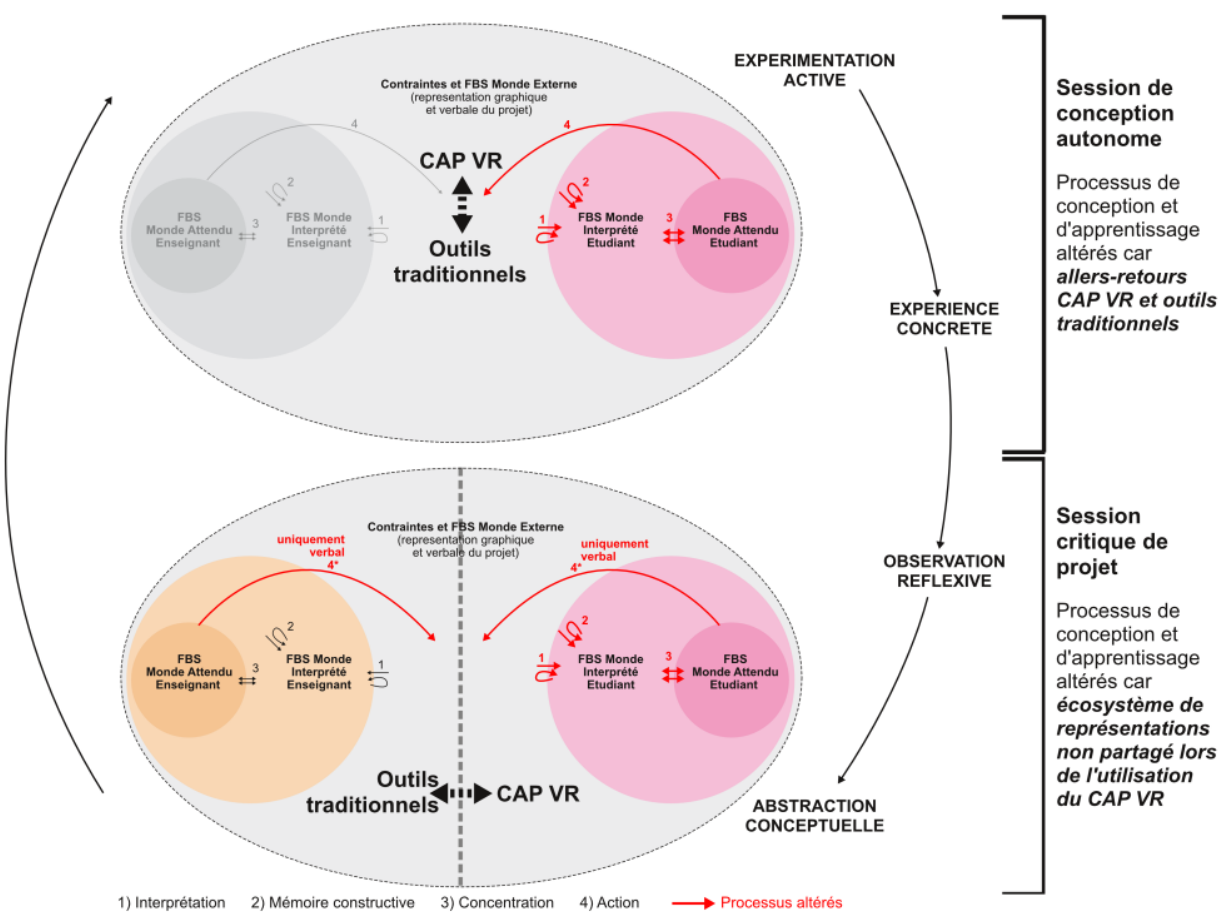

(a) Processus altérés par l'utilisation du CAP VR

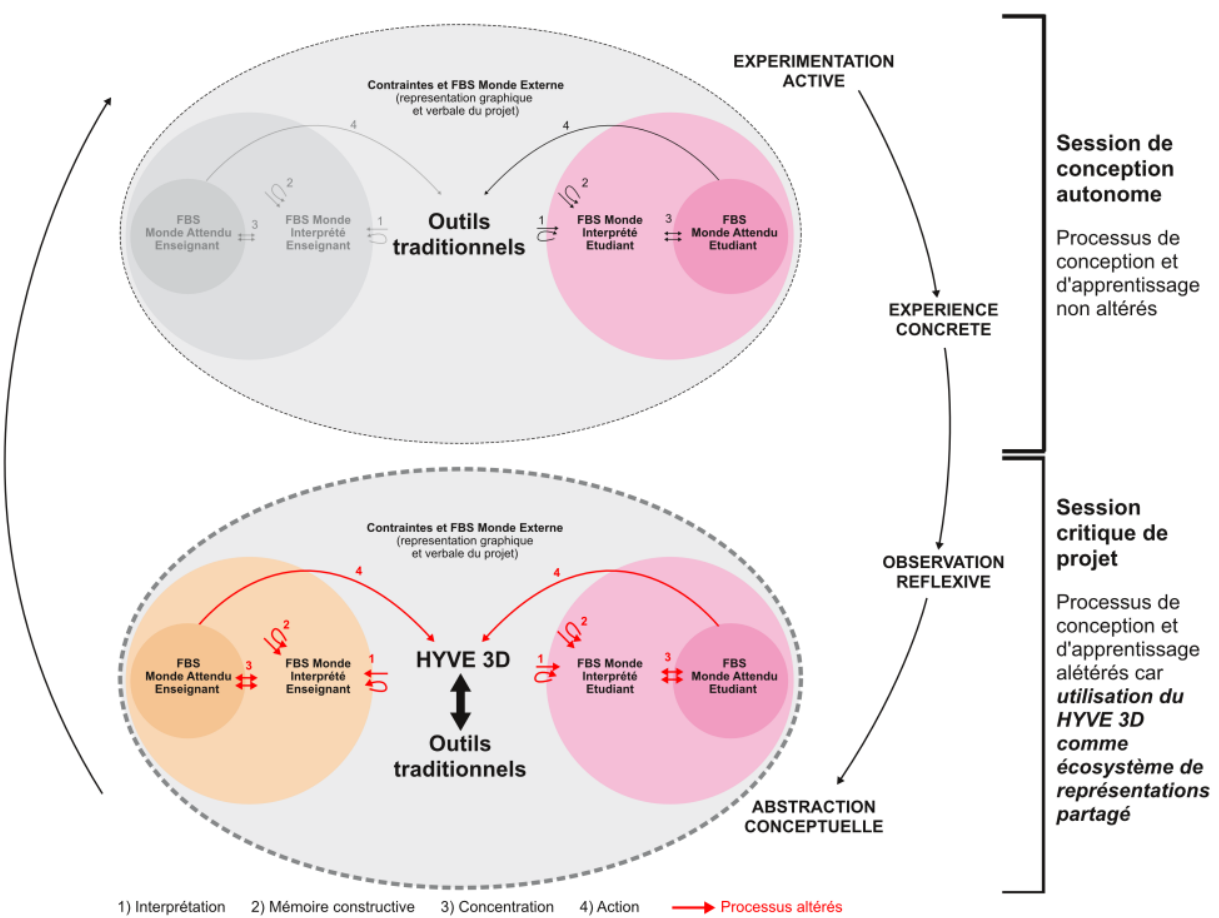

(b) Processus altérés par l'utilisation du HYVE 3D

Figure 4. (a) Implications du CAP VR sur l'apprentissage situé de la conception, (b) Implications du HYVE 3D (b) sur l'apprentissage situé de la conception. 


\section{Discussion}

Nous avons examiné l'implication de l'utilisation de deux écosystèmes de représentation utilisant l'immersion dans un EV comme support d'enseignement de la conception. Dans le cas du CAP VR, l'accent est mis sur les moments d'apprentissage autonomes de l'étudiant par l'augmentation de ses capacités d'auto-évaluation de son projet par le parcours de l'espace du projet en immersion à la première personne. L'évaluation de l'espace par sa taille, proportion, profondeur et densité est renforcée par la visualisation dans le CAP VR (Angulo et de Velasco, 2015). Pour la conception autonome, la boucle de conversation réflexive est plus intense avec le CAP VR que dans un environnement traditionnel et le niveau d'engagement des étudiants dans le processus de conception est amplifié. La qualité du projet est accrue (Angulo, 2015) et l'étudiant se met dans une posture réflexive plus facilement que dans un environnement traditionnel ${ }^{1}$.

L'utilisation du HYVE 3D pour les sessions critiques permet une meilleure prise de décision collaborative car l'espace de représentation hybride est partagé. L'immersion et la perception à l'échelle 1 aide au processus créatif et réduit la difficulté de compréhension de l'espace (Dorta et al., 2016a). Comme les capacités de compréhension de l'espace de l'étudiant sont augmentées, cela favorise une symétrie des actions entre enseignants et étudiants, et renforce les échanges horizontaux entre les participants (Dorta et al., 2012). L'écosystème du HYVE 3D enrichit le codesign, à la fois verbal, gestuel et graphique pendant les sessions. Ces qualités du HYVE 3D, remarquées lors de son utilisation pour des studios de codesign et design industriel à l'Université de Montréal, sont similaires aux retours d'expérience d'étudiants de studio de projet d'architecture (semestre d'hiver 2017, étudiant de l'ENSA Nantes) ayant utilisé le HYVE 3D pour un atelier sur deux jours (LID, Bordeaux). L'immersion dans le projet déplace la relation entre enseignant et étudiants. En effet, le projet fait le cadre, efface le rapport hiérarchique entre les participants et encourage l'échange ${ }^{2}$.

\section{Perspectives}

L'apprentissage de la conception en studio de projet porte des caractéristiques issues de différentes théories sur l'apprentissage (constructivisme, apprentissage social et apprentissage situé). La construction de schémas cognitifs de conception par l'expérience s'accorde avec le format du studio où les étudiants apprennent à concevoir par la pratique du projet. L'enseignant agit comme évaluateur des connaissances de l'étudiant et comme coach, à travers sa critique, pour donner un retour adéquat et personnalisé à chaque étudiant $\mathrm{du}$ studio. L'écosystème de représentations est un acteur important du processus de conception et du processus d'apprentissage de la conception. Il est l'environnement dans lequel les étudiants développent leur expérience individuelle de conception de projet, et il est le support de la discussion durant les sessions critiques, où il acquiert une position d'outil pédagogique. Le cycle d'apprentissage expérientiel de Kolb (1984), l'ontologie de conception située de Gero et Kannengiesser (2004) offrent un cadre propice au développement d'un modèle descriptif des processus d'apprentissage de la conception dans le cadre du studio. Le modèle proposé permet d'illustrer les implications de l'utilisation d'écosystème de représentations utilisant la réalité virtuelle. L'étude de deux dispositifs offrant des systèmes d'immersion distincts, le HYVE 3D avec un écran immersif et le CAP VR avec un casque couplé à un écran vertical, a montré leurs potentiels pour enrichir les

\footnotetext{
${ }^{1}$ Entretien avec Antonieta Angulo le 13/10/2016

${ }^{2}$ Entretien avec les étudiants ayant participés à l'atelier intensif le 11/08/2017
} 
processus d'apprentissage des étudiants. Le CAP VR semble favoriser l'engagement des étudiants dans leur projet, leur apprentissage autonome et leur réflexivité pendant les sessions de studio en présentiel et en dehors. Le HYVE 3D enrichit l'apprentissage par la collaboration et le codesign pendant les sessions critiques en renforçant les échanges horizontaux entre les participants.

La suite de notre travail se focalisera sur l'implémentation d'un écosystème de représentations adapté à la plateforme CORAULIS (Centre d'Observation en Réalité virtuelle Augmentée et Lieu d'Immersion Sonore), décrit dans Milovanovic et al. (2017). Cet écosystème combine une représentation égocentrée sur un écran immersif et une représentation allocentrée à travers une maquette et / ou un plan augmentés. Dans cette configuration, un modèle numérique unique sert de source pour la projection immersive et la projection sur les objets physiques de l'écosystème de représentations par des techniques de Réalité Augmentée Spatiale (Raskar et al., 1998).

\section{Bibliographie}

Adams, R. S., Forin, T., Chua, M. \& Radcliffe, D. (2016). Characterizing the work of coaching during design reviews. Design Studies, 45, 30-67. doi: 10.1016/j.destud.2015.12.007.

Angulo, A. (2015). Rediscovering Virtual Reality in the Education of Architectural Design: The immersive simulation of spatial experiences. Ambiances, (1). doi: 10.4000/ambiances.594.

Angulo, A., \& Vasquez de Velasco, G. (2015). Virtual Sketching: Instructional Low Resolution Virtual Reality Simulations. In Proceedings of the 19th Conference of the Iberoamerican Society of Digital Graphics, 2, 506-513. Florianopolis, Brazil.

Adams, R. S., Forin, T., Chua, M. and Radcliffe, D. (2016). Characterizing the work of coaching during design reviews. Design Studies, 45, 30-67. doi: 10.1016/j.destud.2015.12.007.

Angulo, A. (2015). Rediscovering Virtual Reality in the Education of Architectural Design: The immersive simulation of spatial experiences. Ambiances, (1). doi: 10.4000/ambiances.594.

Bucciarelli, L. L. (2001). Design Knowing \& Learning. In Design Knowing and Learning: Cognition in Design Education. Elsevier, 297-314. doi: 10.1016/B978-0080438689/50013-9.

Callon, M. (1996). Le Travail de Conception En Architecture. Les Cahiers de La Recherche Architecturale, 37, 25-35.

Cross, N. (1982). Designerly Ways of Knowing. Design Studies, 3 (4), 221-227. doi: 10.1016/0142-694X(82)90040-0.

Cross, N. (2007). Forty Years of Design Research. Design Studies, 28 (1), 1-4. doi: 10.1016/j.destud.2006.11.004.

Cross, N. (2006). Designerly ways of knowing. London: Springer. doi: 10.1007/1-84628$301-9$.

Cruz-Neira, C., Sandin, D. J., DeFanti, T. A., Kenyon, R. V., \& Hart, J. C. (1992). The CAVE: Audio Visual Experience Automatic Virtual Environment. Communications of the ACM, 35 (6), 64-72. doi: 10.1145/129888.129892.

Darke, J. (1979). The Primary Generator and the Design Process. Design Studies, 1 (1), 3644. doi: 10.1016/0142-694X(79)90027-9.

Dewey, J. (1938). Experience and Education. New York: Kappa Delta Pi.

Dorst, K., \& Cross, N. (2001). Creativity in the Design Process: Co-Evolution of Problemsolution. Design Studies, 22 (5), 425-37. Doi: 10.1016/S0142-694X(01)00009-6. 
Dorta, T, \& Kinayoglu, G. (2014). Towards a New Representational Ecosystem for the Design Studio. In Proceedings of the 19th International Conference on Computer Aided Architectural Design Research, 699-708. Hong Kong.

Dorta, T, Kinayoglu, G., \& Boudhraâ, S. (2016a). A New Representational Ecosystem for Design Teaching in the Studio. Design Studies, 47, 164-186. doi: 10.1016/j.destud.2016.09.003.

Dorta, T., Kinayoglu G., and Hoffmann, M. (2016b). Hyve-3D and the 3D Cursor: Architectural Co-Design with Freedom in Virtual Reality. International Journal of Architectural Computing, 14 (2), 87-102. doi: 10.1145/2785585.2785586.

Dorta, T., Lesage A., \& Di Bartolo, C. (2012). Collaboration and Design Education Through the Interconnected HIS: Immature vs. Mature CI Loops Observed Through Ethnography by Telepresence. In Proceedings of the 30th ECAADe Conference, 97105. Prague, Czech Republic.

Dorta, T., \& Pérez, E. (2006). Immersive Drafted Virtual Reality. In Proceedings of the 25th Annual Conference of the Association for Computer-Aided Design in Architecture, 304-316. Louisville, Kentucky.

Gero, J. S. (1990). Design Prototypes: A Knowledge Representation Schema for Design. AI Magazine 11 (4), 26-36. doi: 10.1609/aimag.v11i4.854.

Gero, J S., \& Kannengiesser, U. (2004). The Situated Function-behaviour-structure Framework. Design Studies, 25 (4): 373-91. doi: 10.1016/j.destud.2003.10.010.

Hergenhahn, B. R., and Olson, M. (1997). An Introduction to Theories of Learning. Prentice Hall (5th edition).

Khorshidifard, S. (2011). A Paradigm in Architectural Education: Kolb's Model and Learning Styles in Studio Pedagogy. In ARCC Conference Repository. 621-634.

Kolb, A., \& Kolb, D. (2005). Learning Styles and Learning Spaces: Enhancing Experiential Learning in Higher Education. Academy of Management Learning and Education, 4 (2), 193-212. doi: 10.5465/amle.2005.17268566.

Kolb, D. (1984). Experiential Learning: Experience as the Source of Learning and Development. NJ: Prentice Hall: Englewood Cliffs.

Lawson, B. (2004). Schemata, Gambits and Precedent: Some Factors in Design Expertise. Design Studies, 25 (5), 443-57. doi: 10.1016/j.destud.2004.05.001.

Lawson, B., \& Dorst, K. (2009). Design Expertise. Architectural Press. Routledge.

Lebahar, Jean-Charles. 1983. Le Dessin d'architecte. Simulation Graphique et Réduction d'incertitude. Parenthèses. Roquevaire, France.

Maher, M. L., \& Poon, J. (1996). Modeling design exploration as co-evolution. ComputerAided Civil and Infrastructure Engineering, 11(3), 195-209. doi: 10.1111/j.14678667.1996.tb00323.x

Milovanovic, J., Moreau, G., Siret, D., \& Miguet, F. (2017). Virtual and Augmented Reality in Architectural Design and Education: An Immersive Multimodal Platform to Support Architectural Pedagogy. In Proceedings of the 17th International Conference on Computer Aided Architectural Design, 513-532. Istanbul, Turkey.

Parnell, R, Sara, R., Doidge, C., \& Parsons, M. (2007). The Crit: An Architecture Student's Handbook. 2nd ed. Seriously Useful Guides. Elsevier : Architectural Press.

Phillips, D. C., \& Soltis, J. F. (2009). Perspectives on Learning. Teacher College Press. Fifth Edition.

Piaget, J. (1975). L'équilibration des structures cognitives: problème central du développement. Presse Universitaire de France.

Raskar, R., Welch, G., \& Fuchs, H. (1998). Spatially augmented reality. In First IEEE Workshop on Augmented Reality (IWAR'98), 11-20.

Schön, D. (1983). The Reflective Practitioner: How Professionals Think in Action. London: Temple Smith. 
Schön, D. (1985). The Design Studio. London: RIBA.

Schön, D. (1992). Designing as reflective conversation with the materials of a design situation. Research in Engineering Design, 3 (3), 131-147. doi: 10.1007/BF01580516. 